\title{
Labyrinthe
}

$24 \mid 2006$ (2)

Faut-il être postcolonial?

\section{Des études (post)coloniales à la française}

\section{Grégoire Leménager}

\section{OpenEdition}

\section{Journals}

Édition électronique

URL : http://journals.openedition.org/labyrinthe/1251

DOI : $10.4000 /$ labyrinthe. 1251

ISSN : 1950-6031

Éditeur

Hermann

Édition imprimée

Date de publication : 15 juin 2006

Pagination : 85-90

ISBN : 2-9526131-1-7

\section{Référence électronique}

Grégoire Leménager, « Des études (post)coloniales à la française », Labyrinthe [En ligne], 24 | 2006 (2),

mis en ligne le 25 juillet 2008, consulté le 19 avril 2019. URL : http://journals.openedition.org/

labyrinthe/1251 ; DOI : 10.4000/labyrinthe.1251 


\title{
DES ÉTUDES (POST)COLONIALES À LA FRANÇAISE*
}

\author{
Grégoire LEMÉNAGER \\ gregoirelemenager@yahoo.fr
}

«Il est aujourd'hui devenu difficile d'ignorer la "postcolonialité", tant elle porte des tensions extraordinairement fortes [dans la société française]», affirme-t-on sur le seuil de La Fracture coloniale (p. 10-11). Sans doute. Mais il semble également devenu difficile d'ignorer cet ouvrage collectif paru à l'automne 2005, tant la surexposition médiatique dont il a bénéficié a fait paraître ses auteurs comme les pionniers, en France, d'études coloniales et postcoloniales ${ }^{1}$. Vouées à éclairer le rapport de la société française contemporaine avec son passé colonial, les contributions rassemblées par Pascal Blanchard, Nicolas Bancel et Sandrine Lemaire se trouvent significativement distribuées en deux grandes parties: «Histoire coloniale et enjeux de mémoire» d'une part; «République, "intégration" et postcolonialisme» d'autre part. Chacun des textes mériterait probablement un examen particulier. Cependant, précisons-le d'emblée, il ne s'agit pas ici de rendre compte de l'ensemble de ces contributions, mais bien plutôt de s'interroger sur le cadre général dans lequel s'inscrit cet échantillon d'études (post)coloniales à la française, en focalisant l'attention sur le discours tenu par ses coordinateurs.

\footnotetext{
* Note sur La Fracture coloniale. La société française au prisme de l'héritage colonial, Pascal Blanchard, Nicolas Bancel, Sandrine Lemaire (éd.), Paris, La Découverte, 2005, 312 p., $20 €$.

1. La Fracture coloniale se présente en effet comme un condensé des orientations les plus visibles récemment prises par l'historiographie française dans ce domaine. Ainsi, l'ouvrage s'inscrit directement dans le prolongement d'autres travaux signés ou cosignés par ses coordinateurs et certains de ses auteurs, comme N. Bancel, P. Blanchard et F. Vergès, La République coloniale. Essai sur une utopie, A. Michel, 2003 ; P. Blanchard et S. Lemaire, Culture coloniale, Autrement, 2003, et Culture impériale, Autrement, 2004 ; N. Bancel, P. Blanchard, G. Boëtsch, E. Deroo, S. Lemaire, Zoos humains. Au temps des exhibitions humaines, La Découverte/«Poches», 2004. Autour de P. Blanchard semble ainsi s'être constitué un véritable groupe, auquel La Fracture coloniale ajoute d'autres noms, comme ceux de Benjamin Stora, Marc Ferro (co-auteur du Livre noir du colonialisme, Robert Laffont, 2004), ou encore Olivier Le Cour Grandmaison (auteur de Coloniser. Exterminer. Sur la guerre et l'État colonial, Fayard, 2005).
} 
D'un point de vue théorique, ce cadre semble posé dès l'introduction: «À la vérité, nous n'inventons rien. Depuis plus de deux décennies, la littérature scientifique de langue anglaise, dans les postcolonial studies, les subaltern studies mais aussi certains aspects des french studies, ont fait de la question postcoloniale (postcolonial theory) un champ historique pleinement légitime» (p. 13). Autrement dit, La Fracture coloniale serait là pour accuser et rattraper un retard de la recherche hexagonale sur la recherche anglo-saxonne, en naviguant dans son sillage: idée qui revient encore sous la plume de Nicolas Bancel, lorsque celui-ci déplore «la faible visibilité internationale de la recherche française dans le champ des colonial studies et des postcolonial studies ${ }^{2} »$. L'indistinction règne, cependant, dans la rapide énumération inaugurale des studies qui fleurissent sur les campus américains. Et du groupe d'historiens et sociologues réunis dans cet ouvrage, on pourrait donc attendre qu'ils se situent dans le champ épistémologique vaste et touffu dont ils se réclament.

Or, il apparaît rapidement que leur objet n'est pas là. Ainsi, les références bibliographiques sont rares, et souvent limitées aux titres des quelques ouvrages ayant fait l'objet d'une traduction française : si le nom d'Edward W. Said est cité dans l'introduction ${ }^{3}$, on peut s'étonner dans l'ensemble de l'ouvrage de l'absence d'un nom désormais aussi célèbre, outre-Atlantique, que celui de Gayatri C. Spivak. Mais surtout, les auteurs de l'introduction admettent volontiers leur peu d'intérêt pour les débats conceptuels suscités, ailleurs, par «le terme "postcolonial"». Parce qu' «il est difficile de donner une définition précise de ce qu'il recouvre», c'est dans leur acception la plus sommaire que se trouvent - brièvement - invoquées les postcolonial studies: «[Elles] ouvrent un champ où sont pensées les articulations colonisation/postcolonisation dans les pays ex-colonisés tout autant que dans les ex-métropoles » (p. 13). Le questionnement théorique se trouve ainsi vite évacué, au profit d'une pratique de l'histoire qui vise beaucoup plus fondamentalement un horizon politique. C'est en effet là que semble être la priorité. «Penser les articulations colonisation/immigration» (p. 26) est l'un des enjeux majeurs de l'entreprise - comme le confirme le titre de la seconde grande

2. Voir «L'histoire difficile : esquisse d'une historiographie du fait colonial et postcolonial», p. 83.

3. Et encore ce nom de Said apparait-il assez curieusement comme celui de l'auteur de Culture et Impérialisme, là où l'on attendrait davantage la référence à son ouvrage majeur, considéré comme fondateur des études postcoloniales : L'Orientalisme. L'Orient créé par l'Occident [1978], Paris, Seuil, 1980. 
partie: «République, "intégration" et postcolonialisme». Le «concept» de «fracture coloniale» renvoie autant à un clivage entre passé et présent qu'aux failles fissurant actuellement une société française confrontée au problème aigu de l'immigration : «concept» pragmatique, si l'on ose dire, car forgé pour examiner de façon urgente cette société française, «au prisme» d'un «héritage colonial» dont elle ignore «aveuglément» les «réalités » et, par conséquent, les «effets ». L'enjeu n'est donc pas ici d'élaborer une théorie postcoloniale, mais bien d'exhumer des faits historiques et sociaux méconnus, longtemps demeurés dans l'ombre de l'histoire nationale.

Cette mise en évidence inaugurale d'une «longue occultation» des questions coloniales et postcoloniales, dans les institutions universitaires et scolaires, constitue assurément l'une des lignes de force du livre. Sur le passé colonial d'Haïti, comme sur ce «drame périphérique» que constitue encore pour nous la guerre d'Algérie, ou sur d'autres «points aveugles » de l'enseignement scolaire ${ }^{4}$, il s'agit de combler d'inquiétants «trous de mémoire » (p. 47 et 57), de mettre fin à un «impensé colonial » (p. 118) produit par des mécanismes de «refoulement» et de «dénégation» (p. 59) typiquement français: faisant le jeu d'une sorte de «révisionnisme colonial», «une partie des historiens [auraient] préféré favoriser le "deuil" à l'histoire» (p. 16). D'un texte à l'autre, le lexique utilisé n'est pas indifférent sur un tel sujet. Mais il ne s'agit guère, à ce niveau, de s'interroger à l'aide de schèmes psychanalytiques sur l'identité hybride des acteurs pris dans le jeu des relations (post)coloniales - ni donc de mobiliser les travaux d'un Frantz Fanon sur la question, par exemple. C'est bien plutôt une psychanalyse collective, brutale mais salutaire, que nous prescrit La Fracture coloniale. Le terme récurrent de l'introduction l'indique expressément : le « déni» (p. 14, 24, 26 et 29) du passé colonial de la République ne peut que s'accompagner d'un dangereux « retour du refoulé» (p. 10), dont les symptômes avant-coureurs se font chaque jour plus pressants. Rien de tel, en somme, que la béance creusée par ce déficit d'histoire pour favoriser, dans une logique de surenchère victimaire, le libre déploiement d'une dangereuse «politique de la

\footnotetext{
4. Voir respectivement les contributions de M. Dorigny, «Aux origines : l'indépendance d'Haïti et son occultation», p. 45-55 ; B. Stora, «Quand une mémoire (de guerre) peut en cacher une autre (coloniale)», p. 57-65 ; S. Lemaire, «Colonisation et immigration : des "points aveugles" de l'histoire à l'école?», p. 93-104.
} 
mémoire» (p. 16), se traduisant par «la confrontation de mémoires concurrentes » (p. 10).

Ainsi tracés, les contours de ce «champ de bataille mémoriel» (p. 23) permettent de cerner la cible principale de l'ouvrage: le traditionnel «modèle d'intégration» républicain, qui aujourd'hui «vacille», et que l'entreprise entend dépouiller de son voile de vertus prétendument universalistes. Dès lors, «ouvrir des perspectives authentiquement postcoloniales» (p. 23) doit consister à «poser la question de la diversité et de l'altérité» (p. 25) et à «dépasser les tabous de l'imaginaire colonial» (p. 29) sous la lueur d'une hypothèse clé : celle de «la généalogie républicaine de l'idéologie coloniale moderne» (p. 42). Par-delà les limites chronologiques instituées par les dates de la décolonisation, mettre au jour les «noces historiques entre le fait colonial et la République» impliquerait de considérer comme les fruits de cette union scandaleuse ${ }^{5}$ certains phénomènes sociaux contemporains (i.e. les problèmes liés à l'immigration, principalement). Ce qui revient pour Pascal Blanchard à poser cette question, centrale à ses yeux: «La colonisation est-elle consubstantielle de la République?» (p. 33.)

De telles analyses ne manquent certes pas de cohérence, ni d'une pertinence que tendrait d'ailleurs à valider une série d'événements récents (depuis la revendication des «indigènes de la République» jusqu'aux violences inédites survenues quelques semaines après la parution de La Fracture coloniale dans de nombreuses banlieues françaises, en passant par les vigoureuses protestations suscitées, à retardement, par le désormais fameux article 4 de la loi du 23 février $2005^{6}$ ). On ne peut cependant se défendre d'une certaine perplexité face au volontarisme citoyen qui sous-tend cet ouvrage d'historiens - et semble parfois lui tenir

5. «Il y a évidemment un scandale de la colonisation», affirme Marc Ferro selon lequel «en Indochine, en Afrique, la République a trahi ses valeurs »; ce qui peut apparaître de la part de cet historien comme une façon, prudente et sans doute pertinente, de poser le problème sans amalgamer pour autant universalisme républicain et dérives coloniales. Voir «La colonisation française : une histoire inaudible», p. 130.

6. Rappelons que, dans cette loi du 23 février 2005 «portant reconnaissance de la nation et contribution nationale en faveur des Français rapatriés», l'article 4 disposait que «les programmes de recherche universitaire accordent à l'histoire de la présence française outre-mer, notamment en Afrique du Nord, la place qu'elle mérite» et, de façon beaucoup moins neutre cette fois, que «les programmes scolaires reconnaissent en particulier le rôle positif de la présence française outre-mer, notamment en Afrique du Nord, et accordent à l'histoire et aux sacrifices des combattants de l'armée française issus de ces territoires la place éminente à laquelle ils ont droit...» 
lieu de cadre méthodo-épistémologique. D'une part, l'introduction paraît ouvrir la voie d'une vision «désoccidentalisée» (comme le dirait G. C. Spivak) de l'histoire coloniale et de ses effets: «Il s'agit [...] de passer de l'un au multiple, opération déjà considérable, et qui permettrait de relativiser ce qui est encore ressassé de manière permanente, à savoir le "génie français" et la "mission universelle" de la France, dans le monde métissé qui est désormais le nôtre» (p. 28). Mais d'autre part, la virulente critique du «concept d" "intégration" ", menée ici tambour battant, rend pour le moins équivoques les termes empruntés à l'historien Patrick Weil pour légitimer les travaux rassemblés : «La perception de l'histoire des autres, leur intégration dans l'histoire nationale, c'est l'exigence ultime et nécessaire de la diversité dans la République » (cité p. 27 - nous soulignons). Benjamin Stora ne conclut pas autrement son intéressante contribution: «L'histoire des Maghrébins appartient à l'histoire trop méconnue de la colonisation, qui est pourtant partie intégrante de l'histoire de France» (p. 65 - nous soulignons). Au terme d'une dialectique serrée, une «opération» finale consisterait donc à repasser «du multiple à l'un» pour reconstruire une autre mémoire collective, cette fois commune à tous, et préserver la cohésion nationale en ajoutant à nos manuels d'histoire de France quelques chapitres inédits: "Notre approche, dans le prolongement de nos travaux récents, vise à replacer le passé colonial de la France dans la pensée et l'historiographie nationales, afin de produire des perspectives rendant intelligibles les situations postcoloniales» (p. 29).

Sans doute la démarche se conçoit-elle de la part de citoyens, militant pour bâtir sans tarder une digue d'histoire afin de contenir «les ressacs de la colonisation» (p. 16) ; mais la perspective de l'historien ne risque-t-elle pas de s'en trouver quelque peu orientée - faussée? Un curieux paradoxe énonciatif, qui n'est peut-être pas si insignifiant qu'il en a l'air, hante en effet le discours de plusieurs auteurs de La Fracture coloniale. Du moins dans les principes et les finalités qu'ils affichent, tout se passe comme si leur attitude venait au fond rédupliquer celle de l'ethnocentrisme républicain dont ils tiennent à prendre le contre-pied ; car malgré toutes les précautions dont elle s'entoure ${ }^{7}$, la revendication

7. «Il y a plusieurs manières d'être nationaliste, et l'extrême droite n'a pas le monopole de cette postur », précise-t-on notamment, lorsqu'il s'agit de légitimer l'importance accordée à «la question nationale», p. 27. 
de «nationalisme», affirmée dans une volonté de «préservation des valeurs nationales» et dans un souci de garantir la cohésion sociale, résonne ici étrangement. Le «prisme de l'héritage colonial», tel qu'il semble adopté, n'est-il pas singulièrement franco-centré? Il semble en tout cas quelque peu contradictoire, et bien périlleux, d'adopter à son tour une conception utilitaire de l'histoire après en avoir si vigoureusement dénoncé le principe.

Que devient dans ces conditions la «décolonisation des imaginaires» préconisée dans l'ouvrage par Patrick Simon (p. 237-246)? Celle-ci pourrait bien achopper sur ce qu'Achille Mbembé désigne à un tout autre niveau comme «l'aporie [qui se trouve] au cœur de la logique de l'intégration et de l'assimilation »: «Le problème de ceux qui, tout en étant avec nous, parmi nous ou à côté de nous, ne sont finalement pas des nôtres» (p. 140). Si, comme le soutient A. Mbembé, la position d' «altérité radicale» assignée aux colonisés (d'Afrique notamment) est un problème lié à «l'impensé de la race», qui n'aurait été «résolu ni par l'abolition de l'esclavage, ni par la décolonisation» (p. 140), on peut se demander si la construction d'un grand récit national multiculturaliste, dans un mouvement d'élargissement du champ de l'historiographie hexagonale visant à «intégrer» le passé des colonisés, ne reviendrait pas à pérenniser le problème d'une autre façon, en objectivant les colonisés dans une nouvelle position d' "altérité radicale». À cet égard, instruire le procès d'une République qui serait coloniale non seulement historiquement mais aussi par essence, comme le propose Pascal Blanchard, comporte un risque qui semble inscrit dans les gènes mêmes de la démarche adoptée par l'historien-citoyen : celui de rejouer l'éternel faceà-face $d u$ colonisateur et $d u$ colonisé, en s'empêchant de penser dans leur diversité les relations et les acteurs que recouvrent et produisent les phénomènes (post)coloniaux. Ainsi, notamment, du rôle d'émancipation qu'ont aussi pu jouer les valeurs universalistes de la République auprès des colonisés - sinon de la possibilité pour les anciens colonisés de s'approprier, à leur tour, ces valeurs. 